\title{
MUSCLE FUNCTION IN THE FINGERS
}

\author{
H. Graham Stack, London, England
}

From the Albert Dock Fracture and Orthopaedic Hospital, London

The functions of the muscles and tendons that move the fingers have been discussed for many years, and many actions have been ascribed to the different parts of the system.

Duchenne (1867) thought that the interossei extended the interphalangeal joints, and that the extensor communis was responsible for extension of the metacarpo-phalangeal joint. Sunderland (1945) wrote that the muscles function as well integrated and coordinated groups in every movement of the digits. The importance of synergism was emphasised by Bunnell (1942) who believed that the lumbricals and interossei have different actions in the various stages of movement, and he explained this by what he called the shift of the aponeurotic sleeve. This idea can be extended to other muscles, which have different actions and points of action for almost every stage of movement.

The present study is based on the generally accepted details of anatomy, mainly as expounded by Bunnell (1948), Landsmeer (1949, 1955), Haines (1951) and Eyler and Markee (1954), and on the electro-myographic studies of Backhouse and Catton (1954). It is supported by several new observations, and the deductions are explained with the help of working models.
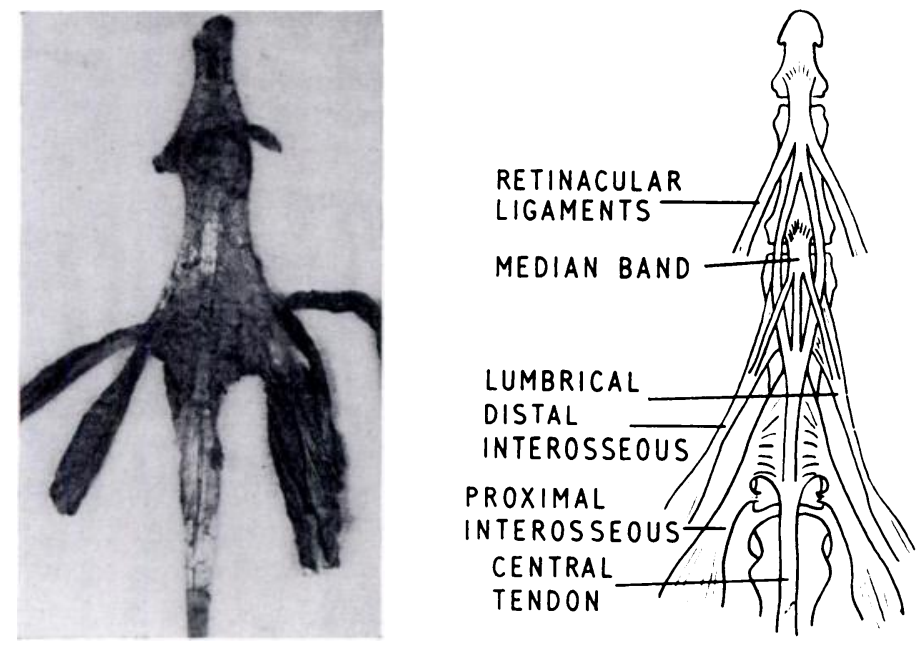

Fig. 1

A photograph of the extensor assembly, with a diagram.

THE EXTENSOR EXPANSION

Landsmeer (1949) gave a new description of the extensor expansion, showing it to be a plexus of tendons rather than a sheet of tendon tissue. These tendons are thought to have more freedom within the expansion than is suggested by previous descriptions. The basis of the assembly is the central tendon, which divides into three (Fig. 1). The central, intermediate or median band-which Landsmeer called the medial band-is attached to the base of the middle phalanx (Grant 1958). The two lateral bands rejoin beyond this to form the terminal tendon, and this is attached to the dorsum of the distal phalanx.

This system is joined by three pairs of components. 1) Distally, it is joined by the oblique parts of the retinacular ligaments of Landsmeer (1955). These arise from the flexor tendon sheath on the proximal phalanx, run in front of the proximal interphalangeal joint, and over 


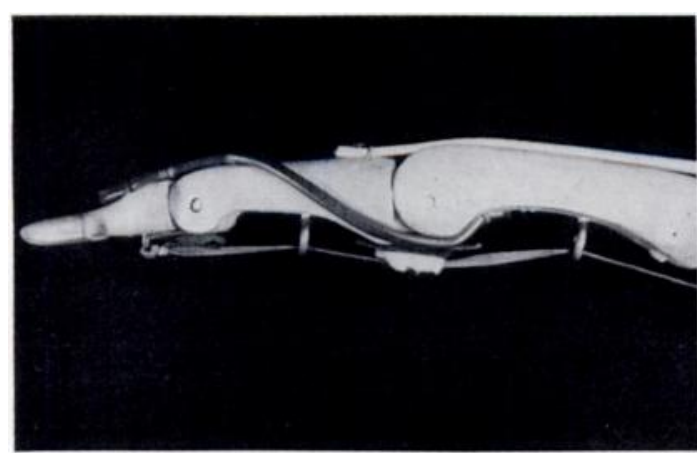

Fig. 2

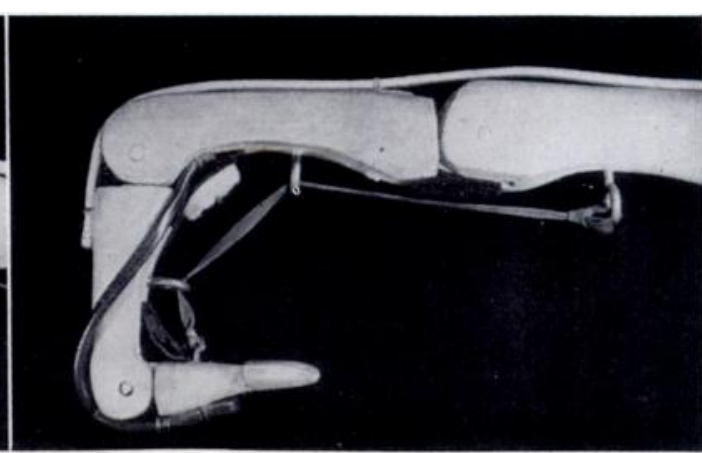

Fig. 3

A model to show that the retinacular ligament links the movements of the two distal joints transmitting the pull of the median band to the distal phalanx. (The model has joints with central axes.)

the side of the middle phalanx, to join the terminal tendon behind the distal interphalangeal joint. Functionally they link the movement of the two joints, so that normally they are always in the same degree of flexion. 2) Intermediately, the wing tendons (Landsmeer 1955) join the basic assembly from either side of the finger. The details of the muscles serving them are complicated, but it is usually a lumbrical on the radial side and a palmar interosseous on the ulnar side. Each tendon is inserted in two parts. The superficial parts (the spiral fibres) join the median band of the central tendon to be inserted into the base of the middle phalanx. The deep parts fan out to be inserted into the lateral bands of the central tendon. 3) Proximally, the system is joined by the so-called phalangeal tendons. These usually arise from a dorsal

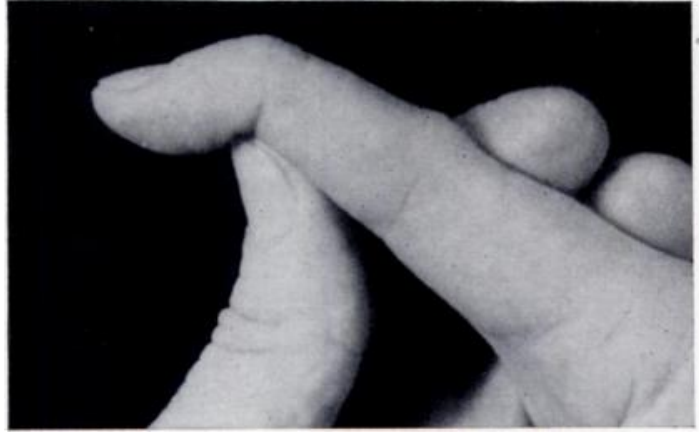

FIG. 4

To show that, when the proximal interphalangeal joint is held forcibly fully extended, there is sufficient laxity in the retinacular ligament to allow about 45 degrees of flexion in the distal interphalangeal joint. interosseous muscle, and also have a bifid insertion (Grant 1958), first to the phalangeal tubercle at the base of the proximal phalanx, and second, through a fan of tendinous fibres, to the central tendon. Taken all together this junction forms the expansion or " dossiere" (Bunnell 1942).

In general, the assembly moves as one piece, as described by Bunnell (1942), but the arrangement of the tendons and of the intertendinous fibres allows of some relative movement between the tendons. Bunnell himself, quoting Hauck (1923), pointed out that the lateral and volar movement of the lateral tendons was to allow the flexion of both interphalangeal joints for the same excursion of the central extensor tendon. For this to occur there must be some freedom of movement between the lateral and median tendons, and therefore the tissue between them must to some extent be elastic.

The attachment of the muscles by bifid tendons allows them to have different points of action in different positions of the hand, when the line of force is applied at one or other of the attachments, or at a point between the two.

The physiology of the system has been made much clearer by the electro-myographic studies of Backhouse and Catton (1954), who showed that action currents in the lumbricals are present only when the interphalangeal joints are in extension. Action currents are present in the interossei in both flexion and hyperextension of the metacarpal joints, as well as in abduction movements. 


\section{THE RETINACULAR LIGAMENTS}

Landsmeer (1955) postulated that the arrangement of the retinacular ligaments maintains the constant angles of flexion between the interphalangeal joints, and transmits the pull of the median extensor tendon to the distal phalanx.

This is demonstrated by the model shown in Figures 2 and 3. The interphalangeal joints are constructed with fixed axes, the central extensor tendon is represented by a single strand attached to the base of the middle phalanx, and the retinacular ligament runs from the tendon sheath to the dorsum of the distal phalanx. A rubber band provides the flexor mechanism.

On pulling and relaxing the extensor tendon, the middle phalanx is extended and flexed, and at the same time the retinacular ligament transmits the pull to the base of the distal phalanx, and the distal interphalangeal joint is extended and flexed. The two joints move in concert and are always at the same angle.

But if in the living hand a proximal interphalangeal joint is held firmly by the other hand in the position of full extension, the distal joint can be flexed, usually to about 45 degrees. Theoretically, if the retinacular ligaments were taut this would be impossible (Fig. 4).

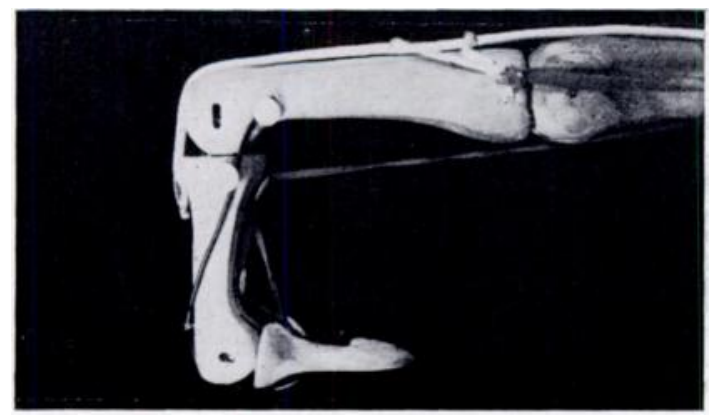

FIG. 5

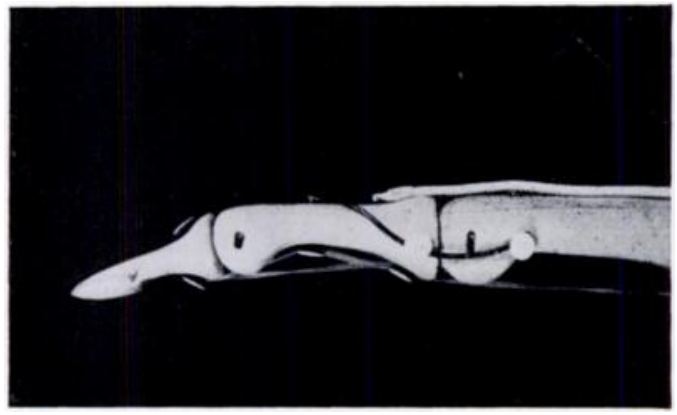

FiG. 6

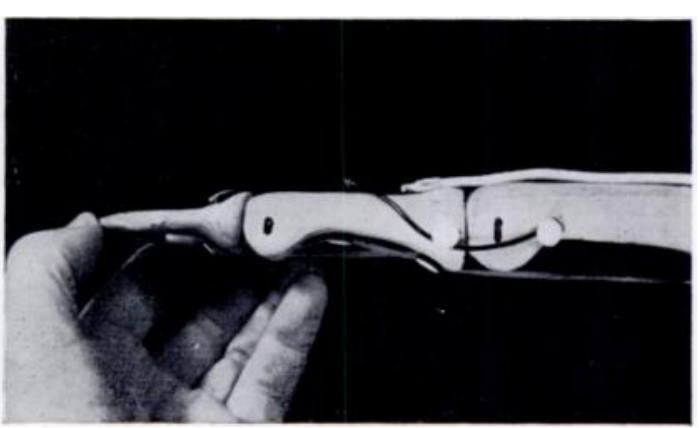

Fig. 7

The joints in this model are constructed with movable axes which slide forward in flexion. The retinacular ligament is tight in flexion and relaxed in extension; another extending system is required to achieve full extension of the distal joint.

\section{THE INTERPHALANGEAL JOINTS}

The interphalangeal joint surfaces are eccentric, in that there is a move volarwards of the axes of flexion during the movement of flexion. This means that the axial length of the bones of the finger is longer in flexion than in extension; the retinacular ligaments are, therefore, tighter in flexion than in extension. The relaxation in extension means that the retinacular ligaments are unable to complete the movements of full extension at the distal interphalangeal joint, for which another system is required.

The second model demonstrates these points (Figs. 5 to 7). The axes of the joints in this model consist of a nail running in a slot, and, as the joints flex, this nail moves volarwards.

VOL. 44 B, NO. 4, NOVEMBER 1962 
In the more flexed positions the angle between the bones at both joints remains about the same, but as extension is approached the distal phalanx lags behind, and, at full extension of the proximal joint, the distal joint is still not fully extended.

The wing tendons-The eccentricity of the interphalangeal joint surfaces also increases the tendency for the tension of the wing tendons to be applied, in the flexed position, through the spiral fibres to the base of the middle phalanx, instead of to the lateral tendons. They assist by extending the proximal joint at the beginning of the movement from full flexion, and only affect the distal joint indirectly through the retinacular ligaments.

In the latter half of extension this tension is applied to the lateral tendons, and helps in extending the distal interphalangeal joint directly. The considerations immediately below apply only to the position of nearly full extension.

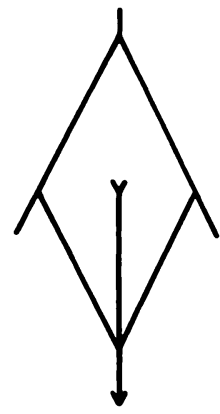

FIG. 8

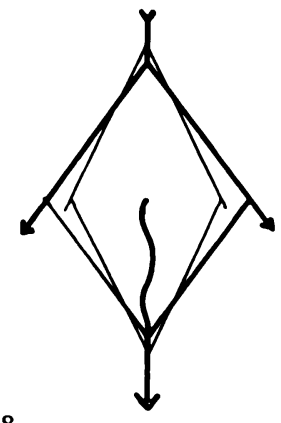

A diagrammatic representation of the diamond of tendons made by the extensor expansion over the proximal interphalangeal joint. The force of the wing tendons, applied at the angles, tends to widen and shorten the diamond, and partially to relax the median band.

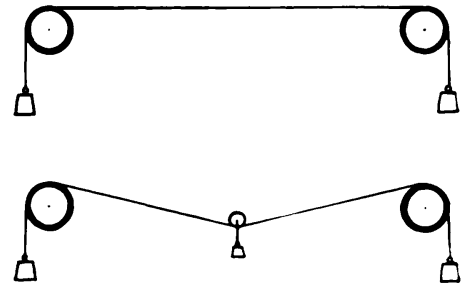

FIG. 9

To show how a small weight applied in the centre of a system will alter the line of the tension holding the bigger weights.

The lateral extensor tendons-The arrangement of these tendons can be considered as a diamond with the central tendon proximally, the phalangeal attachment distally, and the wing tendons coming in at an angle of 25 degrees at the other two corners (Fig. 8). The force of the wing tendons can be resolved into a pure extending force acting at the distal attachment, and two opposite forces at right angles to it, which widen and shorten the diamond. Thus the wing tendons tense the lateral tendons by pulling them sideways and forwards over the base of the middle phalanx and the head of the proximal phalanx.

It was in order to explain this mechanism that the separate movements of the median and lateral tendons in flexion of the proximal interphalangeal joint were emphasised. It is considered that this sideways movement of the lateral tendons takes place in full extension as well as in full flexion.

The median tendon attached to the base of the middle phalanx is relaxed to some extent by this mechanism, and some of the tension of the long extensor tendon is transmitted to the distal attachment.

An experiment can be performed with a piece of string, with an equal weight on each end, running over two pulleys which are fixed at the same level some distance apart (Fig. 9). When a smaller weight is applied to the centre of the string it descends, and both the larger weights are lifted a little. The tension of the string remains at approximately the value of the larger weights, but this larger amount of tension is transmitted round a longer path, by the application of the smaller weight.

Thus, in full extension of the distal phalanx the main extension force is supplied by the long extensor, but its point of application is altered by a tiny lumbrical or interosseous muscle. It can be likened to a Servo brake mechanism in a car, in which light pressure on the brake pedal causes power from the engine to apply the brakes strongly. 
The next model (Figs. 10 and 11) shows that, although the central tendon alone cannot extend the distal joint, it can do so when the lateral tendons are put under tension. The pull of the long extensor is then transmitted round the lateral tendons, and the median tendon is relatively relaxed.

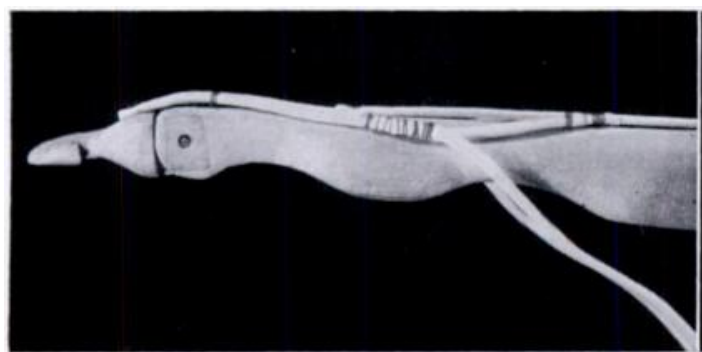

Fig. 10

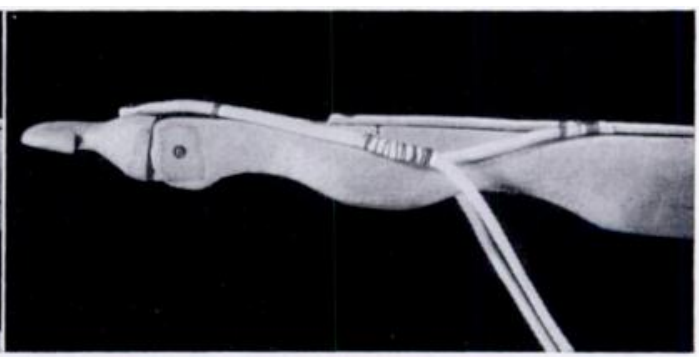

Fig. 11

A small force applied to the wing tendons causes the pull of the long extensor to be transmitted to the distal phalanx, and to produce extension of the distal joint.

\section{THE LUMBRICAL MUSCLES}

Backhouse and Catton's (1954) observation that there were action currents present in the lumbricals only in the presence of interphalangeal extension suggested that it might be valuable to work out the relative lengths of the muscles in the various positions of the fingers.

The finger has two systems of movement: flexion and extension of the metacarpophalangeal joint, and flexion and extension of both the interphalangeal joints together. In order to simplify the calculations, four positions are defined as follows (Fig. 12):

1

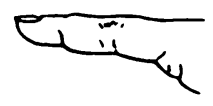

2

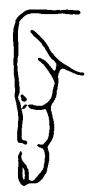

3

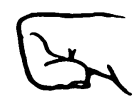

4

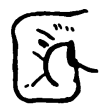

Fig. 12

To show the four basic positions of the fingers.

\section{Full extension}

$\{$ Metacarpo-phalangeal extension. Interphalangeal extension.

2. The lumbrical position. Intrinsic plus $\{$ Metacarpo-phalangeal flexion. Interphalangeal extension.

3. The hook grip. Intrinsic minus $\left\{\begin{array}{l}\text { Metacarpo-phalangeal extension. } \\ \text { Interphalangeal flexion. }\end{array}\right.$

4. Full flexion $\left\{\begin{array}{l}\text { Metacarpo-phalangeal flexion. } \\ \text { Interphalangeal flexion. }\end{array}\right.$

In Figure 13 the lumbrical muscles are represented in each of the four positions. The end on the right is the origin on the flexor tendons, and the end on the left the point at which the tendon starts distally. Beside it, for comparison, is a similar diagram of the distal and proximal interossei.

The diagram was constructed using the following figures of excursions of the tendons, after Bunnell (1948), Kaplan (1953) and Landsmeer (1955). 


\author{
Profundus tendon \\ Metacarpo-phalangeal joint, 1.5 centimetres \\ proximally on flexion. \\ Proximal interphalangeal joint, 2 centimetres \\ proximally on flexion. \\ Distal interphalangeal joint, 0.5 centimetre \\ proximally on flexion.
}

\section{Lumbrical tendon}

Pulley moves 1.2 centimetres proximally on flexion.

Tendon moves 0.9 centimetres proximally on extension.

Tendon movement does not affect the diagram.

Several estimates of the movement of the lumbrical tendon and the distal attachments have been used in working out this diagram, and the present ones are considered the most accurate. However, any alteration in these figures is not very significant, and makes little difference to the final picture, which takes this form because the lumbricals cross from the flexor to the extensor surface, and which is dominated by the enormous proximal movement of the flexor profundus tendon.

The actions of the lumbrical muscles-The lumbricals and the interossei acting on ulnar wing tendons are capable of weak extension of the proximal and distal interphalangeal joints by

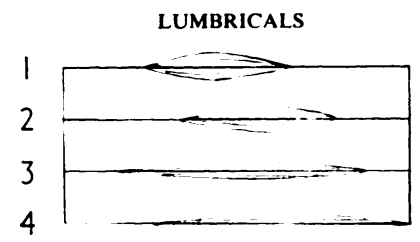

Fig. 13

Lengths of the intrinsic muscles in the four positions shown in Figure 12. Note the progressive proximal movement of the flexor tendon attachments of the lumbrical muscles. Note also that there is very little alteration of length in the movements 1 to 2, and 3 to 4 , the movements of flexion of the metacarpophalangeal joint. The distal interosseous muscles are the motors for the distal wing tendons. These muscles have fixed origins, but the distal insertions have the same excursions as the lumbrical muscles. The proximal interosseous muscles also have a fixed origin, and the tendon excursions are very similar to those of the distal interosseous muscles. Note that in all three sets of muscles the greatest shortening occurs in the movement 3 to 2, the classical movement of the upstroke in writing. 2 is the position of " intrinsic plus," or of intrinsic contracture. 3 is the position of "intrinsic minus." This is the position that it is impossible to obtain in Parkes's test (1945) in cases of intrinsic contracture.

themselves, as in radial palsy. But the full power is only developed when the intrinsics and the long extensors work together-as in a Servo mechanism. The main power of extension is supplied by the long extensor, and its point of application is determined by the intrinsics.

Extension of interphalangeal joints-It will be seen from Figures 13 to 17 that the two positions, 1 and 2 , in which the lumbrical is contracted, are those in which the interphalangeal joints are extended, and that the muscle does not shorten when the metacarpo-phalangeal joint is flexed in the movement 1 to 2 .

In order to hold the interphalangeal joints extended in both extension and flexion of the metacarpo-phalangeal joints, with the same length and power of contraction, the lumbrical must be able to act from a different position, and this it does by having a moving origin from the flexor profundus tendon.

Some tension in the flexor profundus is necessary for the lumbrical to be able to act effectively. In radial palsy, if the proximal phalanges are supported in extension the lumbricals and interossei are capable of producing interphalangeal extension. When the flexor profundus is also paralysed they are not able to carry out this movement so well.

Metacarpo-phalangeal flexion-The movement 1 to 2 represents metacarpo-phalangeal flexion in the presence of interphalangeal extension. With full metacarpo-phalangeal extension, action of the interossei will produce hyperextension; therefore they cannot initiate flexion.

The motor for metacarpo-phalangeal flexion is the flexor digitorum profundus, its tendon travelling proximally by 1.5 centimetres. Because the interphalangeal joints remain extended, 


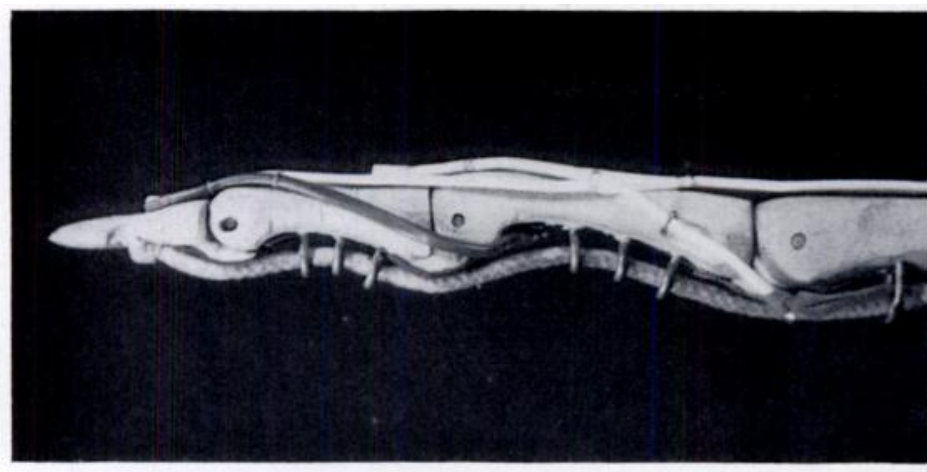

FIgs. 14 AND 15

Figs. 14 To 17

A model is shown in these four basic positions. The lumbrical muscle is short and contracted in Figures 14 and 15, with interphalangeal extension; and is long and relaxed in Figures 16 and 17, with interphalangeal flexion. Its length does not alter with metacarpo-phalangeal movement.

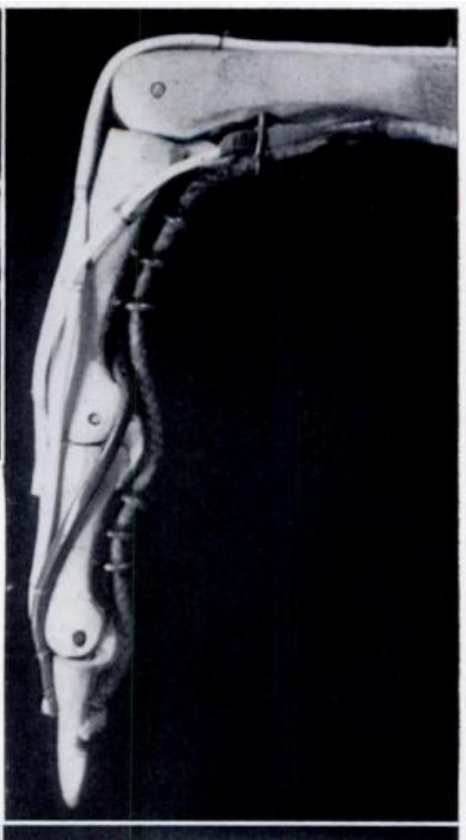

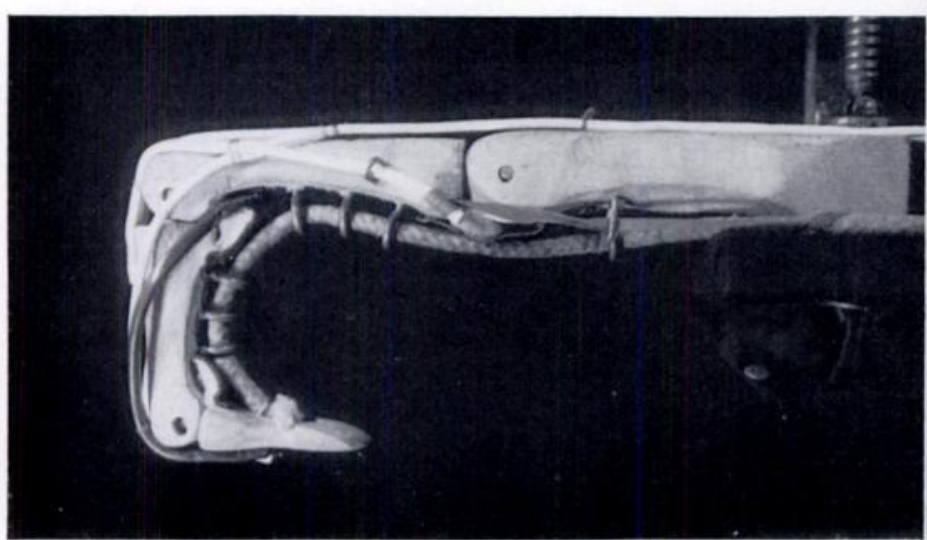

FIG. 16

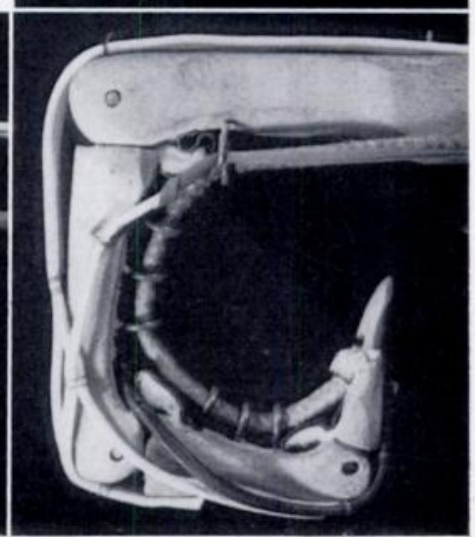

FIG. 17

the distal point of action of the flexor profundus is no longer on the flexor surface of the distal phalanx, but is transferred, by the tension of the lumbrical, to the extensor expansion. The flexor tendon beyond the origin of the lumbrical does not partake in the movement, during which the lumbrical itself remains contracted - and under tension-without altering its length. The distal movement of the flexor tendons-The action of extension of the interphalangeal joints in the two positions of the metacarpo-phalangeal joints is represented by the move from the positions 3 to 1 , and 4 to 2 . In both these actions the centre of the lumbrical muscle stays approximately in the same place, and its two ends are approximated. It will also be seen that there is a distal movement of the flexor tendons, carrying the origin of the lumbrical in both these positions.

Needle threading, and the upstroke in writing - These two traditional movements are represented by the move from position 3 to 2 in Figure 13, and here too there is shortening of the lumbrical, indicating active contraction.

Extensible nerve ending - It has been suggested that the lumbrical may act as an extensible nerve ending, to coordinate the movements between the long extensors and flexors. This is supported by the work of Winckler and Rabischong (1961), quoted by Verdan and Michon

VOl. 44 B, NO. 4, NOVEMBER 1962

$K_{(8)}$ 
(1961) who have shown that the lumbricals contain numerous nerve end organs, particularly distally.

\section{THE INTEROSSEOUS MUSCLES}

The classical division of the interossei was into palmar and dorsal, whose actions were considered to be adduction and abduction respectively, in addition to their action of flexion at the metacarpo-phalangeal joint.

They are now regarded as having two types of attachment: first, those going to join the distal wing tendons near the head of the proximal phalanx, most of which are palmar muscles; and second, those joining the assembly near the base of the proximal phalanx. These are attached by a bifid tendon, part to the phalangeal tubercle at the base of the bone and part to the transverse fibres of the " dossiere."

It might be more correct to call them proximal and distal interossei, as it is their insertion rather than their origin which dictates their actions. The dorsal interossei mostly have the proximal attachments, and the palmar mostly have the distal attachments.

The distal interossei-The functions of the distal interossei are very similar to those of the lumbricals, as they are the muscles usually serving the ulnar wing tendons, to pair with the lumbrical on the other side.

Because the interossei do not have moving origins the pattern of their change in length in the various movements differs from that of the lumbricals, and this difference may help to make the movements smoother (Fig. 13).

It should be noted that the greatest shortening lies between the positions 3 and 2; this is the traditional action of the intrinsics, the upstroke in writing. There is also some shortening in the movements of flexion of the metacarpo-phalangeal joints from 1 to 2 and 3 to 4 .

Note also that position 2, in which both the lumbricals and the interossei are at their shortest, is the position of the hand in contracture of these muscles (Parkes 1945, Bunnell 1953). The proximal interossei-It is mostly the dorsal interossei that have a proximal insertion. Their attachment is to an aponeurosis, which lies between the metacarpal heads, and which divides into two parts to be attached respectively to the phalangeal tubercle at the base of the proximal phalanx, and to the proximal wing of the "dossiere." At this point the fibres blend, or are even continuous with those of the opposite side (Fig. 1).

This arrangement allows an alternative point of action of the muscle in different positions of the hand. Simply stated, the phalangeal attachments are used in extension of the interphalangeal joints, and the wing attachments are in use in flexion of these joints (Figs. 18 to 21). This is the shift of the aponeurotic sleeve of Bunnell (1948).

It should be noted that this system would be impossible if the transverse metacarpal ligaments (the deep transverse ligaments of the palm) lay between the metacarpal heads instead of anteriorly, because then they would interfere with the movements of the sling.

In position 1, or extension at both the metacarpo-phalangeal and the interphalangeal joints, the wing attachments are relaxed, all the tension is applied at the phalangeal attachments, and abduction or adduction is produced. This obviates misapplication of the tension on the extensor tendon, which otherwise might be dislocated. It can be seen that, in adduction or abduction of the index finger in full extension, there is no tendency for the long extensor tendon to move from side to side, whereas if the same movement is carried out in flexion of the metacarpo-phalangeal joints, the tendon moves appreciably from side to side.

In position 2 , or flexion of the metacarpo-phalangeal joints, the phalangeal attachments are relaxed and all the tension of the proximal interossei is applied to the expansion; this gives an excellent moment for flexion, but adduction and abduction are more difficult.

In position 3, with metacarpo-phalangeal extension-or even with hyperextension-and interphalangeal flexion, the point of action of the muscle is divided between the two attachments, and the line of pull is thus carried dorsally behind the attachment to the phalangeal 
tubercle, so increasing the moment exerted by the muscle behind the axis of the metacarpophalangeal joint. This explains why the action of hyperextension is so much stronger in this position than it is in the presence of interphalangeal extension, when the line of pull is not altered.

Backhouse and Catton (1954) have noticed that there are action currents in the interossei in the normal action of hyperextension of these metacarpo-phalangeal joints, and Braithwaite, Channell, Moore and Whillis (1948) pointed out that in burns involving the dorsum of the

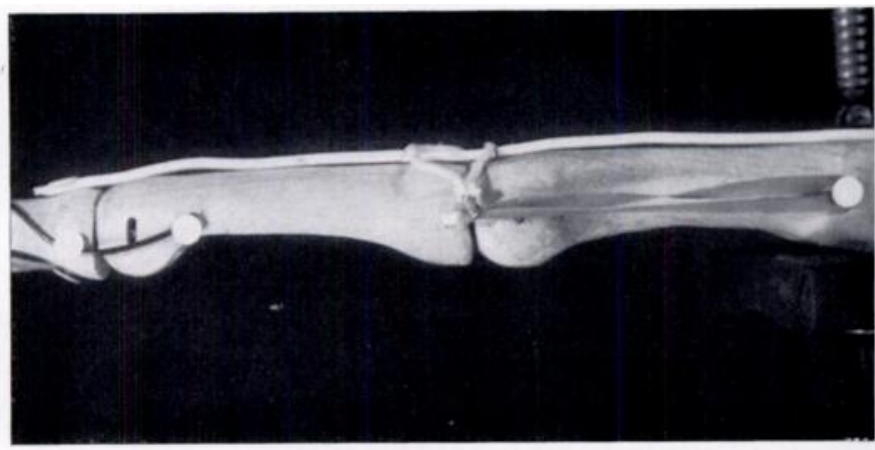

FIGS. 18 AND 19

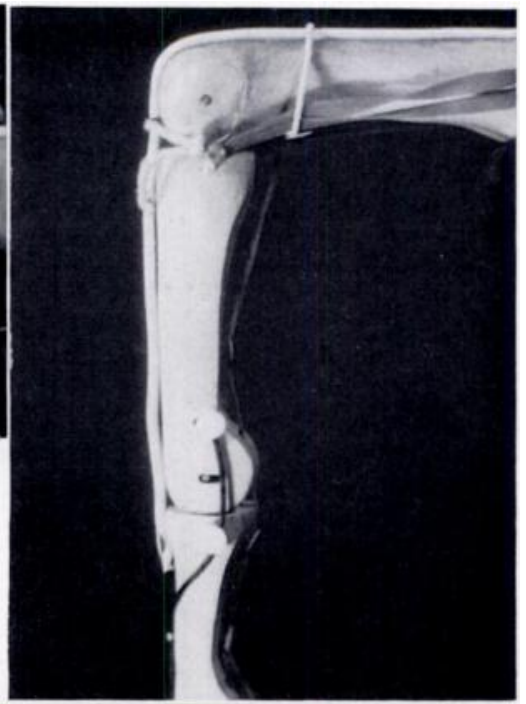

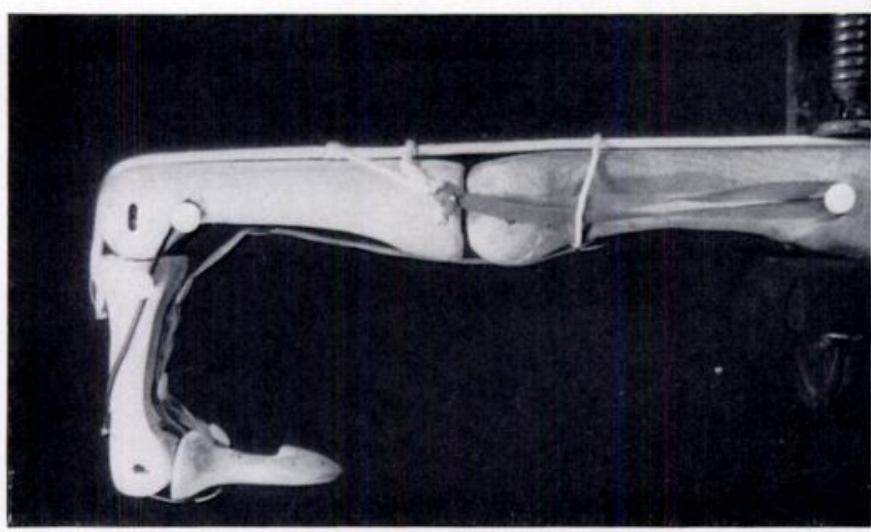

FIG. 20

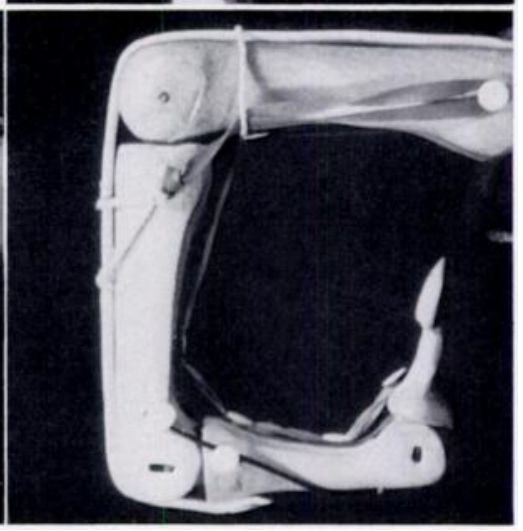

FIG. 21

A model showing the proximal interosseous with its sling attachment, in the four positions. In Figure 18, when it is on the line of the axis, it produces no flexion or extension, but only movements of adduction or abduction. In Figure 19 it is anterior to the axis, and produces flexion. There is little tension transmitted to the extensor tendon, thus reducing the chance of dislocation of the tendon. In Figure 20, with the pull divided between the phalangeal attachment and the extensor tendon-and behind the axis-extension, or even hyperextension, is produced. Finally, in Figure 21, the pull is divided between the phalangeal tubercle and the extensor expansion - and in front of the axis-and strong flexion is produced, because the long extensor is not in action, and all the tension is applied to the sling round the bone.

hand in which there is hyperextension, the interossei-which would normally be expected to flex the metacarpo-phalangeal joints-iend to worsen the hyperextension. They also pointed out that the actions of adduction and abduction and flexion at the metacarpo-phalangeal joints can be combined in the movement of digital rotation, which is necessary to modify the position of the fingers in the various modifications of the precision grip described by Napier (1956). The lumbrical takes no part in this.

VOL. 44 B, NO. 4, NOVEMBER 1962 
In position 4, with full flexion of all joints, the point of attachment to the "dossiere" moves forwards, thus giving both attachments a moment greater than the phalangeal attachment alone could supply, to give the strongest grip, as well as firm stabilisation of the extensor tendons.

The actions of the intrinsic muscles can te tabulated as in Table I.

There is a great economy of muscle bulk in the hands, because the same muscles can be used for varying actions.

TABLE I

ACtion OF THE INTRINSIC Muscles OF THE HAND

\begin{tabular}{|c|c|c|}
\hline $\begin{array}{l}\text { Position of fingers } \\
\text { (see Figure 12) }\end{array}$ & $\begin{array}{l}\text { Proximal } \\
\text { interossei }\end{array}$ & $\begin{array}{l}\text { Lumbricals and } \\
\text { distal interossei }\end{array}$ \\
\hline $1\left\{\begin{array}{l}\text { Metacarpo-phalangeal extension } \\
\text { Interphalangeal extension }\end{array}\right.$ & $\begin{array}{l}\text { Abduction or } \\
\text { adduction }\end{array}$ & $\begin{array}{l}\text { Interphalangeal } \\
\text { extension }\end{array}$ \\
\hline $1\left\{\begin{array}{l}\text { Metacarpo-phalangeal hyperextension } \\
\text { Interphalangeal extension }\end{array}\right.$ & Hyperextension & $\begin{array}{l}\text { Interphalangeal } \\
\text { extension }\end{array}$ \\
\hline $2\left\{\begin{array}{l}\text { Metacarpo-phalangeal flexion } \\
\text { Interphalangeal extension }\end{array}\right.$ & Flexion & $\begin{array}{l}\text { Interphalangeal } \\
\text { extension } \rightarrow \text { metacarpo- } \\
\text { phalangeal flexion }\end{array}$ \\
\hline $3\left\{\begin{array}{l}\text { Metacarpo-phalangeal extension } \\
\text { Interphalangeal flexion }\end{array}\right.$ & $\begin{array}{l}\text { Weak abduction } \\
\text { or adduction }\end{array}$ & No action \\
\hline $3\left\{\begin{array}{l}\text { Metacarpo-phalangeal hyperextension } \\
\text { Interphalangeal flexion }\end{array}\right.$ & Hyperextension & No action \\
\hline $4\left\{\begin{array}{l}\text { Metacarpo-phalangeal flexion } \\
\text { Interphalangeal flexion }\end{array}\right.$ & Flexion & No action \\
\hline
\end{tabular}

\section{CLAWING IN ULNAR PALSY}

Like the movements of the fingers this deformity can be divided into two parts: hyperextension at the metacarpo-phalangeal joints and flexion at the interphalangeal joints. Hyperextension at the metacarpo-phalangeal joint-When the intrinsic muscles are paralysed the metacarpo-phalangeal joints cannot be stabilised or flexed by the proximal interossei, nor can the distal interossei carry out flexion.

When the lumbricals are out of action the flexor digitorum profundus loses its attachment to the wing tendons, and therefore is unable to carry out its action either of flexing the metacarpo-phalangeal joint, or of assisting to extend the interphalangeal joints. Thus the long extensor is unopposed at the metacarpo-phalangeal joint, and produces hyperextension.

In the position of clawing, the long extensor acts on its attachment to the base of the middle phalanx because the slight flexion of the proximal interphalangeal joint relaxes the insertion into the base of the distal phalanx.

Flexion at the distal joints--In the normal action of full extension at all joints when the lumbrical is in action there must be some tension in the profundus tendon, and this tension is transmitted by the lumbrical to the extensor expansion. When the lumbrical is paralysed, this tension is transmitied beyond the origin of the lumbrical muscle, and flexes the terminal joints until the retinacular ligaments become tense.

In high ulnar palsy the long flexors are also paralysed and the only muscle in action is the long extensor which, acting on the extensor expansion alone and with no opposition, is capable of producing a considerable amount of extension. 


\section{SUMMARY}

1. The extensor assembly of the fingers consists of the central tendon joined by three pairs of components: a) the retinacular ligaments, which link the movements of the interphalangeal joints $; b$ ) the " wing " tendons, a lumbrical on the radial side, and usually a palmar interosseous on the ulnar side; $c$ ) the phalangeal tendons, usually dorsal interossei.

2. The retinacular ligaments are relaxed in full extension of the proximal interphalangeal joints and are, in this position, unable to extend the distal joints fully. This is because the interphalangeal joint surfaces are eccentric.

3. The pull of the wing tendons alters the shape of the extensor expansion and transfers the pull of the long extensor tendon from the base of the middle phalanx to the base of the distal phalanx, thus enabling full extension of the distal joint to be powerfully achieved.

4. The action of the lumbrical muscle, as an extensor of the interphalangeal joint, is demonstrated by a diagram showing its site and length in the various positions of the finger, calculated from the known excursions of the tendons. This is consistent with the observations on action potentials.

5. The phalangeal tendons of the dorsal interossei have a bifid insertion, $a$ ) into the phalangeal tubercle at the base of the proximal phalanx, and $b$ ) into the transverse band, and hence to the central tendon. The muscle acts at one or both of these attachments, according to the positions of the metacarpo-phalangeal and interphalangeal joints, in its varying functions of flexion, abduction and hyperextension.

Finally an explanation of the deformity of clawing in ulnar palsy is given.

\section{REFERENCES}

BACK house, K. M. (1960): Digital Rotation. Journal of Anatomy, 94, 453.

Back house, K. M., and CAtton, W. T. (1954): An Experimental Study of the Functions of the Lumbrical Muscles in the Human Hand. Journal of Anatomy, 88, 133.

Braithwaite, F., Channell, G. D., Moore, F. T., and Whillis, J. (1948): The Applied Anatomy of the Lumbrical and Interosseous Muscles of the Hand. Guy's Hospital Reports, 97, 185.

Bunnell, S. (1942): Surgery of the Intrinsic Muscles of the Hand other than those Producing Opposition of the Thumb. Journal of Bone and Joint Surgery, 24, 1.

Bunnell, S. (1948): Surgery of the Hand. Second edition. Philadelphia and London: J. B. Lippincott Company.

Bunnell, S. (1953): Ischaemic Contracture, Local, in the Hand. Journal of Bone and Joint Surgery, 35-A, 88.

Duchenne, G. B. A. (1867): Physiologie des Mouvements. English edition translated by E. B. Kaplan. Philadelphia: W. B. Saunders Company, 1959.

Eyler, D. L., and MARKeE, J. E. (1954): The Anatomy and Function of the Intrinsic Musculature of the Fingers. Journal of Bone and Joint Surgery, 36-A, 1.

Grant, J. C. Boileau (1958): A Method of Anatomy. Sixth edition. London: Baillière, Tindall \& Cox Ltd.

HaINES, R. W. (1951): The Extensor Apparatus of the Finger. Journal of Anatomy, 85, 251.

Hauck, G. (1923): Die Ruptur der Dorsalaponeurose am ersten Interphalangealgelenk. Archiv für Klinische Chirurgie, 123, 197.

Kaplan, E. B. (1953): Functional and Surgical Anatomy of the Hand. Philadelphia: J. B. Lippincott Company.

LANDSMeER, J. M. F. (1949): The Anatomy of the Dorsal Aponeurosis of the Human Finger and its Functional Significance. Anatomical Record, 104, 31.

LANDSMeER, J. M. F. (1955): Anatomical and Functional Investigations on the Articulation of the Human Fingers. Acta Anatomica, Supplementum 24.

NAPIER, J. R. (1956): The Prehensile Movements of the Human Hand. Journal of Bone and Joint Surgery, 38-B, 902.

Parkes, A. R. (1945): Traumatic Ischaemia of Peripheral Nerves with Some Observations on Volkmann's Ischaemic Contracture. British Journal of Surgery, 32, 403.

Sunderland, S. (1945): The Actions of the Extensor Digitorum Communis, Interosseous and Lumbrical Muscles. American Journal of Anatomy, 77, 189.

Verdan, C., and Michon, J. (1961): Le traitement des plaies des tendons fléchisseurs des doigts. Revue de Chirurgie Orthopédique, 47, 285.

Winckler, G., and Rabischong, P. (1961): Personal communication to Verdan, C., Michon, J., and Stack, H. G.

VOL. 44 B, NO. 4, NOVEMBER 1962

$$
\mathrm{K}-1_{(8)}
$$

